\title{
Contributions of higher partial waves to the elastic scattering amplitude for various long-range interactions
}

\author{
J. M. Wadehra and Sultana N. Nahar \\ Department of Physics and Astronomy, Wayne State University, Detroit, Michigan 48202
}

(Received 29 January 1987)

\begin{abstract}
The contributions of higher partial waves to the elastic scattering amplitude are dominated by long-range interactions which fall off as $r^{-\pi}$ as $r \rightarrow \infty$. Closed-form expressions for the contributions of higher partial waves $(2 l>n-3)$ to the scattering amplitude for various long-range interactions $(n$ ranging from 3 to 8 ) are presented.
\end{abstract}

When the interaction between a projectile and a target is central, it is convenient to use the method of partial waves to obtain the elastic scattering amplitude and the corresponding differential and integrated cross sections. Each partial wave corresponds to a definite angular momentum of the system. For a fixed incident energy of the projectile, the higher partial waves correspond to larger impact parameters of the incoming projectile. If the impact parameter is larger than the range of the interaction, the contribution of the corresponding partial wave to the scattering amplitude, for this particular ener$\mathrm{gy}$, is zero. Thus for short-range interactions between projectile and target only a finite number of partial waves make contributions to the scattering amplitude. Moreover, the number of contributing partial waves increases as the impact energy increases. If the projectile-target interaction has a long-range tail, then, in principle, an infinite number of partial waves will contribute to the amplitude. In practice, the phase shifts of the first few (say, $L$ ) partial waves are computed exactly by solving for the asymptotic radial part of the wave function and the phase shifts of the higher partial waves (from $L+1$ to infinity) are estimated by the Born approximation using only the long-range interaction. (For some recent examples see Refs. 1 and 2.) The purpose of this work is to provide closed-form expressions for the contributions of the higher partial waves to the scattering amplitude for various long-range interactions. As far as we are aware, such a closed-form expression has been obtained previously only for the $1 / r^{4}$ potential; ${ }^{3}$ the results for other long-range interactions are new in the present work.

Consider the elastic scattering of a projectile of mass $\mu$ and energy $E=\hbar^{2} k^{2} / 2 \mu$ by a central potential $V(r)$ which results in a change in momentum from $\hbar \mathbf{k}_{i}$ to $\hbar \mathbf{k}_{f}$ for the projectile $\left(k_{i}=k_{f}=k\right)$. The momentum transfer $\hbar \mathrm{q}=\hbar\left(\mathbf{k}_{f}-\mathbf{k}_{i}\right)$ is related to the scattering angle $\theta$ by $q=2 k \sin (\theta / 2)$. For later convenience we define a quantity $z$ as $z=\sin (\theta / 2)=q / 2 k$. The partial-wave expansion for the elastic scattering amplitude can be written as

$$
f(\theta)=\frac{1}{k} \sum_{l=0}^{\infty}(2 l+1) T_{l} P_{l}(\cos \theta),
$$

where the transition matrix elements $T_{l}$ are related to the phase shifts $\delta_{l}$ by

$$
T_{l}=\exp \left(i \delta_{l}\right) \sin \left(\delta_{l}\right)
$$

If the quantities $T_{l}$ for $l>L$ are approximated by $T_{1 B}$ which are obtained by using the Born approximation, then $f(\theta)$ can be rewritten as

$$
f(\theta)=\frac{1}{k} \sum_{l=0}^{L}(2 l+1) T_{l} P_{l}(\cos \theta)+\Delta f,
$$

where

$$
\begin{aligned}
\Delta f & =\frac{1}{k} \sum_{l=L+1}^{\infty}(2 l+1) T_{l B} P_{l}(\cos \theta) \\
& =f_{B}(\theta)-\frac{1}{k} \sum_{l=0}^{L}(2 l+1) T_{l B} P_{l}(\cos \theta) .
\end{aligned}
$$

Here $f_{B}(\theta)$ is the complete scattering amplitude in the Born approximation, that is,

$$
\begin{aligned}
f_{B}(\theta) & =-\frac{2 \mu}{\hbar^{2}} \int_{0}^{\infty} d r V(r) r^{2} \frac{\sin (g r)}{q r} \\
& =-\frac{2 \mu}{\hbar^{2}} \int_{0}^{\infty} d r V(r) r^{2} \sum_{l=0}^{\infty}(2 l+1) j j^{2}(k r) P_{l}(\cos \theta) \\
& =\frac{1}{k} \sum_{l=0}^{\infty}(2 l+1) T_{l B} P_{l}(\cos \theta)
\end{aligned}
$$

and $T_{I B}$ is the Born approximation for $T_{l}$, namely,

$$
T_{l B}=-\frac{2 \mu k}{\hbar^{2}} \int_{0}^{\infty} d r V(r) r^{2} j_{l}^{2}(k r) .
$$

Now, since only the long-range part of the interaction contributes to phase shifts of higher partial waves, it is reasonable to take for $V(r)$, in Eq. (5), a general spherically symmetric potential of the form

$$
V(r)=C_{n} a_{0}^{n-1} e^{2} / r^{n} \text {. }
$$

Here $a_{0}=\hbar^{2} / m e^{2}$ is the Bohr radius, $-e$ and $m$ are the charge and mass of an electron, and $C_{n}$ are dimensionless coefficients. For certain values of $n$ the two terms on the right-hand side of Eq. (3c) could be individually singular but the difference, namely, $\Delta f$ is always finite and nonsingular. Substituting from (6) into (5) and using Eq. 6.574.2 of Ref. 4 one obtains 
$T_{l B}=-\frac{C_{n}}{2} \frac{\mu}{m} \sqrt{\pi}\left(k a_{0}\right)^{n-2} \frac{\Gamma\left(l-\frac{n}{2}+\frac{3}{2}\right) \Gamma\left(\frac{n}{2}-\frac{1}{2}\right)}{\Gamma\left(\frac{n}{2}\right) \Gamma\left(l+\frac{1}{2}+\frac{n}{2}\right)}$.

The conditions of validity for this expression are

$$
n>1, l>(n-3) / 2 \text {. }
$$

Table I shows explicit expressions for $T_{1 B}$ for values of $n$ from 3 to 8 . The corresponding long-range interactions, along with their possible applications in atomic and molecular physics, are now discussed individually.

(i) $n=3$. A potential of the form $1 / r^{3}$ appears as the leading retardation term (that is, the effect of the finiteness of the speed of light) for electron-electron interactions. ${ }^{5,6}$ In a different context, when two identical neutral atoms, excited to different degrees, exchange a photon it leads ${ }^{7}$ to a "resonance interaction" of the form $1 / r^{3}$. Using the expression for $T_{\mid B}$ for $n=3$ in Table I and the sum

$$
S_{3}=\sum_{l=1}^{\infty} \frac{(2 l+1) P_{l}(\cos \theta)}{l(l+1)}=-1-2 \ln (z)
$$

where $z=\sin (\theta / 2)$, we obtain the following closed-form expression

$\Delta f=\frac{\mu a_{0} C_{3}}{m}\left[1+2 \ln (z)+\sum_{l=1}^{L} \frac{(2 l+1) P_{l}(\cos \theta)}{l(l+1)}\right]$.

(ii) $n=4$. The case of $1 / r^{4}$ potential, perhaps the most well known in atomic physics, arises as the leading term, in the adiabatic approximation, in interactions of a charged projectile (electron, ion, positron) with a neutral polarizable target. Again using the relevant entry in Table I for $T_{l B}$ and

$$
S_{4}=\sum_{l=0}^{\infty} \frac{P_{l}(\cos \theta)}{(2 l+3)(2 l-1)}=-\frac{z}{2},
$$

the closed form expression for $\Delta f$ becomes

$$
\Delta f=\frac{2 \pi \mu k a_{0}^{2} C_{4}}{m}\left|\frac{z}{2}+\sum_{l=0}^{L} \frac{P_{l}(\cos \theta)}{(2 l+3)(2 l-1)}\right|,
$$

with $z=\sin (\theta / 2)$. This expression has previously been obtained by Thompson. ${ }^{3}$

(iii) $n=5$. When retardation effects are taken into account for interactions between a structureless charged projectile in motion and a neutral polarizable target, the leading correction term behaves ${ }^{8}$ asymptotically as $1 / r^{5}$. The $1 / r^{5}$ retardation effect also appears when applied to an electron-ion system with the electron bound in a high Rydberg state. 910 This term, which is an addition to the $1 / r^{4}$ polarization potential, vanishes in the nonrelativistic limit. Again using the results in Table $I$ and

$$
S_{5}=\sum_{l=2}^{\infty} \frac{(2 l+1) P_{l}(\cos \theta)}{(l+2)(l+1) l(l-1)}=\frac{1}{3}-\frac{z^{2}}{6}+2 z^{2} \ln (z),
$$

the closed form expression for $\Delta f$ in this case can be written as

$$
\begin{aligned}
\Delta f=-\frac{2 \mu k^{2} a_{0}^{3} C_{5}}{3 m} & \mid \frac{1}{3}-\frac{z^{2}}{6}+2 z^{2} \ln (z) \\
& \left.-\sum_{l=2}^{L} \frac{(2 l+1) P_{l}(\cos \theta)}{(l+2)(l+1) l(l-1)}\right),
\end{aligned}
$$

where $z=\sin (\theta / 2)$.

(iv) $n=6$. A long-range potential of the form $1 / r^{6}$ appears in various applications in atomic and molecular physics. First, the nonrelativistic van der Waals interaction between two neutral atoms, each in its lowest energy state, behaves as $1 / r^{6}$ for separations much larger than the Bohr radius. "1 Next, the second term in the interaction energy between a charged projectile and a neutral polarizable target behaves, in the adiabatic approximation, as $1 / r^{6}$. Third, the leading nonadiabatic correction to the dipole term in the interaction between a charged projectile and a neutral polarizable target behaves asymptotically as $1 / r^{6} \cdot 12$ In these cases one can use

$$
S_{6}=\sum_{l=0}^{\infty} \frac{P_{l}(\cos \theta)}{(2 l+5)(2 l+3)(2 l-1)(2 l-3)}=\frac{z^{3}}{18}
$$

and the results of Table I to obtain the following closedform expression for $\Delta f$,

$$
\begin{aligned}
\Delta f= & \frac{6 \pi \mu k^{3} a_{0}^{4} C_{6}}{m} \\
& \times\left[-\frac{z^{3}}{18}+\sum_{l=0}^{L} \frac{P_{l}(\cos \theta)}{(2 l+5)(2 l+3)(2 l-1)(2 l-3)}\right],
\end{aligned}
$$

where, as before, $z=\sin (\theta / 2)$.

TABLE I. Explicit expressions for $T_{l B}$ for various long-range interactions.

\begin{tabular}{llc}
\hline$n$ & \multicolumn{1}{c}{$-\frac{m\left(k a_{0}\right)^{2-n}}{\mu C_{n}} T_{l B}$} & $\begin{array}{c}\text { Condition of } \\
\text { validity }\end{array}$ \\
\hline 3 & $1 /[l(l+1)]$ & $l \geq 1$ \\
4 & $2 \pi /[(2 l+3)(2 l+1)(2 l-1)]$ & $l \geq 1$ \\
5 & $2 /[3(l+2)(l+1) l(l-1)]$ & $l \geq 2$ \\
6 & $6 \pi /[(2 l+5)(2 l+3)(2 l+1)(2 l-1)(2 l-3)]$ & $l \geq 2$ \\
7 & $8 /[15(l+3)(l+2)(l+1) l(l-1)(l-2)]$ & $l \geq 3$ \\
8 & $20 \pi /[(2 l+7)(2 l+5)(2 l+3)(2 l+1)(2 l-1)(2 l-3)(2 l-5)]$ & $l \geq 3$ \\
\hline \hline
\end{tabular}


(v) $n=7$. When retardation effects are taken into account the interaction between two neutral atoms in their lowest energy states behaves as $1 / r^{7}$ for extremely large atom-atom separations. ${ }^{10,13}$ This interaction is a replacement for and not an additive correction to the nonrelativistic van der Waals interaction. Using the appropriate entry from Table I and

$$
\begin{aligned}
S_{7} & =\sum_{l=3}^{\infty} \frac{(2 l+1) P_{l}(\cos \theta)}{(l+3)(l+2)(l+1) l(l-1)(l-2)} \\
& =\frac{43}{240} z^{4}-\frac{z^{2}}{5}+\frac{1}{80}-\frac{z^{4}}{2} \ln (z),
\end{aligned}
$$

the closed-form expression for $\Delta f$ becomes

$$
\begin{aligned}
\Delta f=\frac{8 \mu k^{4} a_{0}^{5} C_{7}}{15 m} & \left(\frac{-43}{240} z^{4}+\frac{z^{2}}{5}-\frac{1}{80}+\frac{z^{4}}{2} \ln (z)\right. \\
& \left.+\sum_{l=3}^{L} \frac{(2 l+1) P_{l}(\cos \theta)}{(l+3)(l+2)(l+1) l(l-1)(l-2)}\right),
\end{aligned}
$$

where $z=\sin (\theta / 2)$.

(vi) $n=8$. In investigations of the Rydberg states of helium it is noted that terms up to $1 / r^{8}$ in the polarization potential arising in the ion-core-electron interaction contribute significantly to the energy levels. ${ }^{14,15}$ With the anticipation that interaction terms behaving asymptotically as $1 / r^{8}$ might also contribute significantly to scattering processes, we provide the following closed-form expression for $\Delta f$ for this case,

$$
\Delta f=\frac{20 \pi \mu k^{5} a_{0}^{6} C_{8}}{m}\left[\frac{z^{5}}{450}+\sum_{l=0}^{L} \frac{P_{l}(\cos \theta)}{(2 l+7)(2 l+5)(2 l+3)(2 l-1)(2 l-3)(2 l-5)}\right]
$$

with $z=\sin (\theta / 2)$, using the results in Table I and the sum

$$
\begin{aligned}
S_{8} & =\sum_{l=0}^{\infty} \frac{P_{l}(\cos \theta)}{(2 l+7)(2 l+5)(2 l+3)(2 l-1)(2 l-3)(2 l-5)} \\
& =-\frac{z^{5}}{450} .
\end{aligned}
$$

The closed-form expressions [Eqs. (9b), (10b), (11b), (12b), (13b), and (14a)] for $\Delta f$ for various long-range potentials depend crucially on the evaluation of sums $S_{i}$, $i=3, \ldots, 8$ [Eqs. (9a), (10a), (11a), (12a), (13a), and (14b)]. These sums can be obtained as follows. On multiplying both sides of the generating function

$$
\sum_{l=0}^{\infty} t^{\prime} P_{l}(\cos \theta)=\left(1-2 t \cos \theta+t^{2}\right)^{-1 / 2}
$$

by $t^{m}$ ( $\left.m=-3,-2,-1,0,1,2,3\right)$ and integrating with respect to $t$ from 0 to 1 , one can establish the following sums:

$$
\begin{aligned}
\sum_{l=0}^{\infty} \frac{P_{l}(\cos \theta)}{(l+4)}= & \left(20 z^{5}-10 z^{4}-\frac{70}{3} z^{3}+10 z^{2}+6 z-\frac{11}{6}\right) \\
& +\left(-20 z^{6}+30 z^{4}-12 z^{2}+1\right) \\
& \quad \times \ln (1+1 / z), \\
\sum_{l=0}^{\infty} \frac{P_{l}(\cos \theta)}{(l+3)}= & \left(-6 z^{3}+3 z^{2}+4 z-\frac{3}{2}\right) \\
& +\left(6 z^{4}-6 z^{2}+1\right) \ln [(1+z) / z], \\
\sum_{l=0}^{\infty} \frac{P_{l}(\cos \theta)}{(l+2)}= & (2 z-1)+\left(-2 z^{2}+1\right) \ln [(1+z) / z], \\
\sum_{l=0}^{\infty} \frac{P_{l}(\cos \theta)}{(l+1)}=\ln [(1+z) / z], & \\
\sum_{l=1}^{\infty} \frac{P_{l}(\cos \theta)}{l}= & -\ln [z(1+z)],
\end{aligned}
$$

$$
\begin{aligned}
\sum_{l=2}^{\infty} \frac{P_{l}(\cos \theta)}{(l-1)}= & \left(2 z^{2}-2 z\right)+\left(2 z^{2}-1\right) \ln [z(1+z)], \\
\sum_{l=3}^{\infty} \frac{P_{l}(\cos \theta)}{(l-2)}= & \left(-7 z^{4}+6 z^{3}+5 z^{2}-4 z\right) \\
& -\left(6 z^{4}-6 z^{2}+1\right) \ln [z(1+z)]
\end{aligned}
$$

where $z=\sin (\theta / 2)$. Now the sums $S_{3}, S_{5}$, and $S_{7}$ can be easily evaluated by first doing the partial fractions of the summand and then using the sums of Eq. (16). The sums $S_{4}, S_{6}$, and $S_{8}$ are evaluated by first doing the partial fractions of the summand, then using

$\left[P_{l+1}(\cos \theta)-P_{l-1}(\cos \theta)\right] /(2 l+1)=\frac{\sin \theta d P_{l}(\cos \theta)}{l(l+1) d \theta}$

[and similar lengthy relations for $\left(P_{l+2}-P_{l-2}\right) /(2 l+1)$ and $\left.\left(P_{l+3}-P_{l-3}\right) /(2 l+1)\right]$ and eventually utilizing the sums of Eq. (16). It is rather remarkable that after many pages of algebra the sums $S_{4}, S_{6}$, and $S_{8}$ turn out to be as simple as in Eqs. (10a), (12a), and (14b), which suggests that there could be an easier and perhaps more general procedure (which obviously eluded us) of evaluating sums of this kind.

Finally we comment that in the present work the contributions of higher partial waves to the elastic scattering amplitude for various long-range interactions are taken into account via the first Born approximation. It is now possible to include, in principle, the additional correction terms via the second Born approximation since an exact analytical expression for the second Born contribution to the transition matrix elements $T_{l}$ for any long-range interaction has been obtained recently by Wadehra. ${ }^{16}$

It is a pleasure to thank Professor Larry Spruch for a helpful conversation. This research has been supported, in part, by the Air Force Office of Scientific Research under Grant No. AFOSR-84-0143. 
${ }^{1}$ R. K. Nesbet and S. Geltman, Phys. Rev. A 33, 3815 (1986).

${ }^{2}$ W. L. van Wyngaarden and $H$. R. J. Walters, J. Phys. B 19, 1817 (1986).

3D. G. Thompson, Proc. R. Soc. London Ser. A 294, 160 (1966).

${ }^{4}$ I. S. Gradshteyn and I. M. Ryzhik, Table of Integrals, Series, and Products (Academic, New York, 1965).

5T. Fulton and P. Martin, Phys. Rev. 95, 811 (1954).

${ }^{6}$ L. Spruch, Phys. Today 39(11), 37 (1986).

${ }^{7}$ H. Margenau and W. W. Watson, Rev. Mod. Phys. 8, 22 (1936).
${ }^{8} \mathrm{~J}$. Bernabeu and R. Tarrach, Ann. Phys. (N.Y.) 102, 323 (1976).

${ }^{9}$ E. J. Kelsey and L. Spruch, Phys. Rev. A 18, 15 (1978).

${ }^{10}$ L. Spruch and E. J. Kelsey, Phys. Rev. A 18, 845 (1978).

${ }^{11}$ F. London, Z. Phys. 63, 245 (1930).

${ }^{12}$ C. J. Kleinman, Y. Hahn, and L. Spruch, Phys. Rev. 165, 53 (1968).

${ }^{13}$ H. B. G. Casimir and D. Polder, Phys. Rev. 73, 360 (1948).

${ }^{14}$ R. J. Drachman, Phys. Rev. A 26, 1228 (1982).

${ }^{15}$ C. K. Au, Phys. Rev. A 34, 3568 (1986).

16J. M. Wadehra, J. Phys. B 19, L761 (1986). 